\title{
Use of Ultrasonic Vibration Instrument for Superficial Parotidectomy, Case Report
}

\author{
Carrillo Rivera Jorge Arnulfo MD ${ }^{1}$, Ferraez Castañeda Esther Diana Carolina MD ${ }^{2}$, Valdez \\ Diaz Brenda $\mathrm{MD}^{3}$, Vallejo Ramirez José Eduardo $\mathrm{MD}^{3}$, Luis Angel Medina Andrade $\mathrm{MD}^{4}$, \\ Josue Castillo Cruz $\mathrm{MD}^{4}$, Gonzalez Bello Javier $\mathrm{MD}^{5}$, Autran Martinez Jorge ${ }^{6}$, Esteban \\ Chaparro Araceli $\mathrm{MD}^{7}$, Rodriguez Rodriguez Carlos Eduardo $\mathrm{MD}^{8}$, Mendez Hernandez \\ Alberto Robles MD $^{8}$, Villanueva Solorzano Pedro Leonardo MD $^{8}$ \\ ${ }^{1}$ Oral and Maxillofacial Surgeon, Hospital General “Dr. Darío Fernández Fierro” ISSSTE. Mexico City \\ ${ }^{2}$ Oral and Maxillofacial Resident, Hospital Regional Lic. Adolfo López Mateos. ISSSTE. Universidad Naval- \\ Escuela de Posgrado Naval, Mexico City, Mexico \\ ${ }^{3}$ Medical Student, Hospital, General De Zona 30, IMSS, Mexico City, Mexico \\ ${ }^{4}$ General Surgery Service, Hospital General de Zona $1{ }^{a}$ Rodolfo Antonio de Mucha Macias, Mexico City, \\ Mexico \\ ${ }^{5}$ Surgical Techniques Professor, Universidad Justo Sierra. \\ ${ }^{6}$ General Estomatologist, Private Practice \\ ${ }^{7}$ Medical Doctor, Hospital General de Mexico, Mexico City, Mexico \\ ${ }^{8}$ Universidad La Salle, Hospital Angeles Metropolitano, Mexico City, México
}

\begin{abstract}
*Corresponding Author: Dr. Jorge Arnulfo Carrillo Rivera. Dental and Maxilofacial Rehabilitation, Consultory 124. Av. Juárez Núm. 8 Col. Las Margaritas, Tlalnepantla Estado de México. Tel 53618677. E-mail: : dr_jorge_carrillo_cmf@hotmail.com
\end{abstract}

\begin{abstract}
Surface parotid lobectomy due to benign or malignant tumors is a procedure that usually requires extensive approaches, with dissection to deep spaces and efficient hemostasis. Ultrasonic vibration instruments dissect and coagulate simultaneously becoming more useful and of frequent use in minimally invasive surgery. It consists of three main components: a generator, and an active tip. The present article mentions a clinical case of superficial parotid lobectomy with an ultrasonic vibration instrument and its advantages.
\end{abstract}

Keywords: Ultrasonic Vibration, Superficial parotidectomy, minimally invasive surgery.

\section{INTRODUCTION}

The minimally invasive surgery is defined as the conjunction of diagnostic and therapeutic techniques for the minimal tissue dissection and better hemostasis, with the goal of diminishing surgical time and secondary effects over the surrounding tissues including metabolic response to trauma.

At the end of the 60s, the ultrasonic vibration was developed for their use in the ophthalmologic surgery, and posteriorly it was adapted to neurosurgery and abdominal surgery. Actually is used in vascular, thoracic, head and neck and abdominal surgeries. ${ }^{1}$

The ultrasonic vibration is used by an instrument that takes advantage of the mechanic energy to produce coagulation of vessels and tissue dissection without the need of an exit source like bipolar energy, allowing their use in patients with pacemaker and implants. ${ }^{2}$

The ultrasonic device produces temperatures about 50 to $100{ }^{\circ} \mathrm{C}$, relatively slow compared with the bipolar electrocautery $\left(100\right.$ to $\left.150^{\circ} \mathrm{C}\right)$. It is constituted by three components, a generator that produces electricity, is transformed to mechanic energy by a system of piezoelectric crystals that vibe with an amplitude between 50 to $330 \mu \mathrm{m}$, and at a constant frequency between 23 to $55.5 \mathrm{kHz}$, the expansion and contraction of this crystals are sent by a handpiece that is dock to the active tip, a scalpel or a scissors. (Figure 1). ${ }^{2,3}$

The ultrasonic coagulation is similar to the electrocautery, however, the mechanism for the 
protein denaturation is different. The electrocautery and surgical laser produce a clot by warming the tissue for protein denaturation. The ultrasonic vibration scissors produce protein denaturalization by the transference of mechanic energy, breaking the tertiary hydrogen bonds. The tissue proteins are transformed to a viscous collagen that seals the small and medium vessels. $^{3,4}$
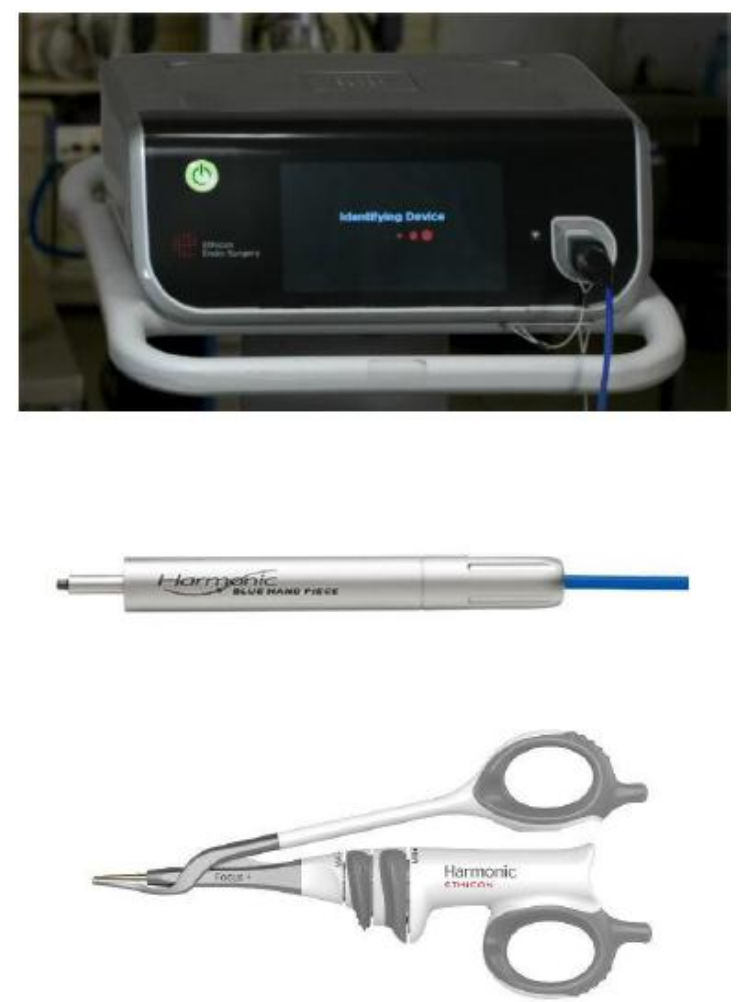

Figure1.Generator, and scissors of the Harmonic Focus $^{M R}$ (Ethicon Jhonson \& Jhonson)

There exist two mechanisms for tissue dissection, one is by cavitational fragmentation and the second is by the mechanical cut at temperatures under $63^{\circ} \mathrm{C}$; the vibration defragments the proteins with the rupture of hydrogen links (tertiary hydrogen links). $3,4,12$

The Harmonic Focus Scissors MR (Ethicon, Jhonson \&Jhonson) can be used simultaneously for cut and coagulation, transferring the neuromuscular electric current with a minimal lateral thermal effect ( 1 to $3 \mathrm{~mm}$ ). After the cut and coagulation, the trans surgery bleeding is minimum, allowing the surgeon to have a better vision during surgery, perform the procedures in a short period of time, and less tissue damage, with a fast recovery, less morbidity, and postoperative pain. ${ }^{9}$

\section{CASE REPORT}

Female patient of 58 years-old with a pathological background of diabetes mellitus 2 .
She refers an increased volume in the left parotid region for two years, with slow growing, firm at palpation, adhered to deep planes, with a diameter of $4 \mathrm{~cm}$., without pain at palpation. An ultrasound reported a hyperechoic zone dependent of the left parotid gland, with adjacent lymph nodes and augmented vascularity. (Figure 2.)

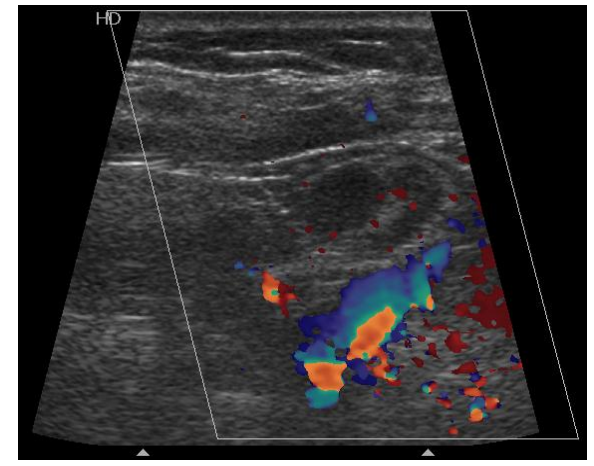

Figure2. Doppler USG of the left parotid region where an augmented vascularity and lymph nodes can be appreciated.

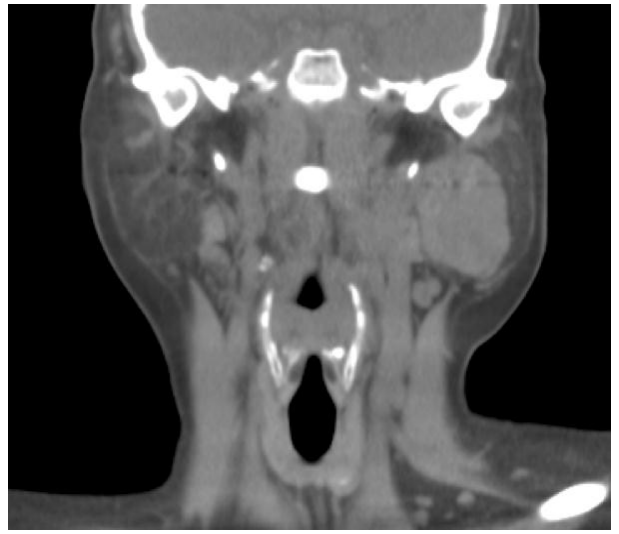

Figure3. Contrasted CT scan in coronal slices where a hyperdense zone in the left parotid region can be appreciated.

Simple and contrasted CT scan is scheduled and reported a hyperdense zone near the carotid sheath and lymph nodes, with a diagnosis of tumor dependent of the parotid gland in their superficial lobe, lymphadenopathy and increased vascularity. (Figure 3.)

A fine needle aspiration was performed and reported polymorphonuclear cells with metaplasia changes in their epithelium, with a diagnosis of Whartin tumor.

Under general anesthesia a submandibular approach was planned, with $3 \mathrm{~cm}$ of length with pre and retroauricular extension, considering Langer lines, the superficial cervical layer was dissected and hemostasis performed at the same time with ultrasonic vibration scissors. A tumor of $4 \mathrm{~cm}$ in the left parotid space was exposed, 
without affection of the marginal branch of the facial nerve and vein. A tumoral resection was performed and sent to pathologic exam confirming the diagnosis of Whartin tumor, without bleeding in surgical site or implants. (Figure 4).

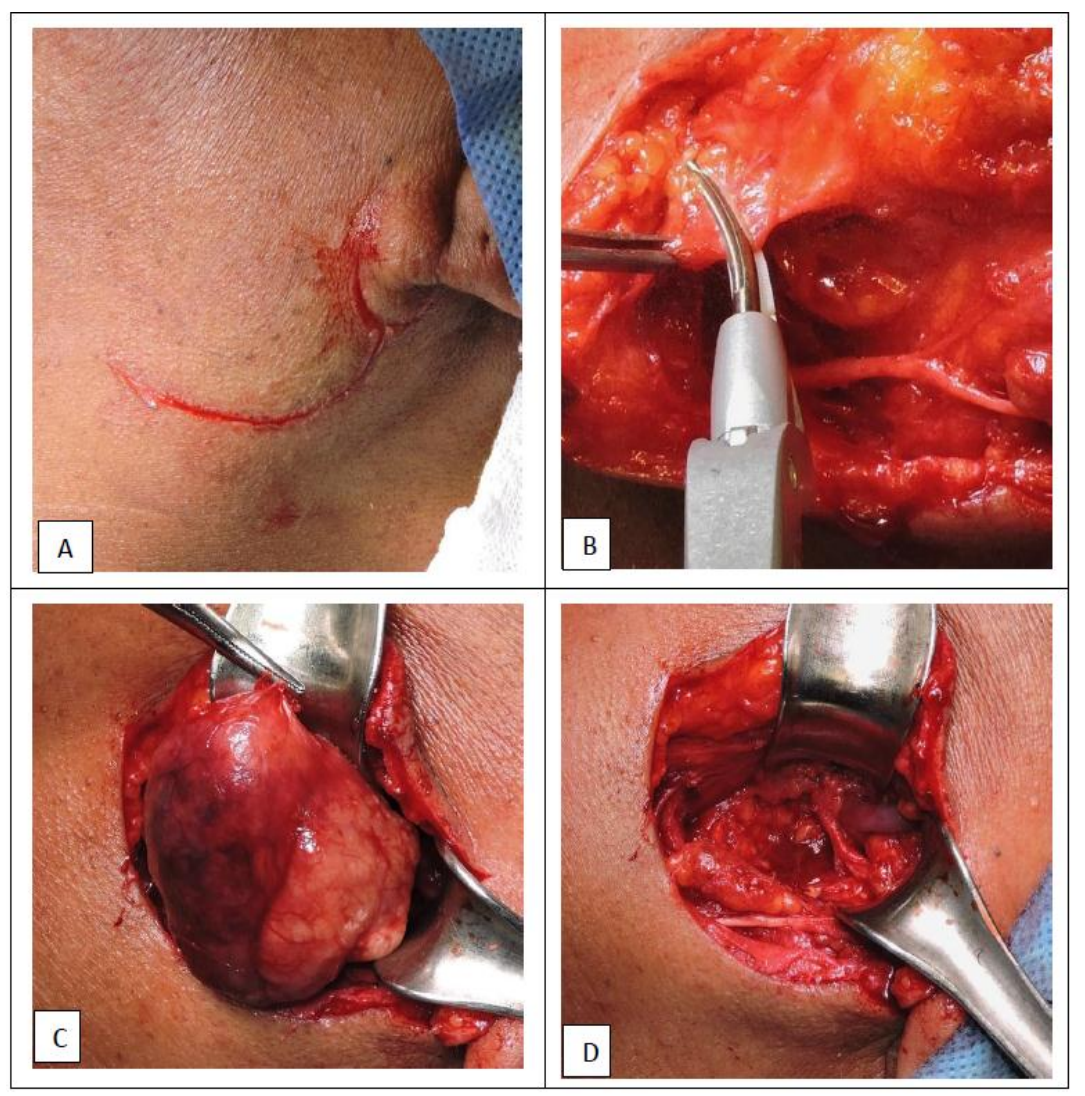

Figure4. Surgical procedure A) Incision design smaller than $3 \mathrm{~cm}$ of length with pre and retroauricular extension. $B$ ) Dissection and coagulation with ultrasonic vibration scissors C) Parotid tumor resection. D) The surgical site with a marginal branch of the facial nerve and facial vein without affection

\section{DISCUSSION}

In 2014, Tirelli et. al. evaluate the advantages and disadvantages associated to the use of ultrasonic vibration in head and neck, with evaluation of surgical time, cost, efficacy, safety, and postsurgical evolution, demonstrating advantages in those areas compared to conventional management. According to this study the use of harmonic ultrasound save 36 minutes for each neck dissection. The trans surgical bleeding was reduced too in neck dissection, oral and oropharyngeal surgery. One mentioned disadvantage was the lymphedema in the neck dissection. ${ }^{4}$

Mathialagan and cols realized a randomized clinical trial, comparing the efficacy of the Harmonic Focus Scissors MR (Ethicon Jhonson\&Jhonson) and the use of electrocautery to avoid the accessory spinal nerve during neck dissection for oral cancer, evaluating the shoulder function and pain in the follow up after surgery, with more incidence of pain and shoulder weakness in the second group inclusive 6 months after surgery. ${ }^{5}$
Song Fan and cols. demonstrated the use of robotic surgery assisted by ultrasonic scissors for the benign lesions resection in the thong base through a transoral approach with a decrease in surgical time and bleeding, and less nerve damage in neck dissections. ${ }^{8 \mathrm{y} 14}$

Verma and Cols. performed a case-control randomized clinical trial in 40 patients with oral cancer that require neck selective dissection and were older than 18 years. The experimental group consisted of 20 patients that underwent selective neck dissection with Harmonic Focus MR (Ethicon Jhonson \& Jhonson), with a total bleeding of $115 \mathrm{ml}$ compared with $127 \mathrm{ml}$ in the other group that used electrocautery. ${ }^{11}$

\section{CONClusions}

The ultrasonic vibration scissors allow the surgeon to produces hemostasis in arterial vessels of $3 \mathrm{~mm}$ and venous vessels of $5 \mathrm{~mm}$ of diameter, with the absence of hematoma or postsurgical bleeding in different studies. ${ }^{4}$ 
The use of ultrasonic dissection instruments for neck dissection is becoming popular after many authors have probed the efficiency and safety of those, with reduction of bleeding and surgical time in neck dissection, thyroid and parathyroid surgeries. ${ }^{5}$

One of the greatest advantages is the reduction of surgical time by diminish the instruments interchange for cut and dissection, reduction of bleeding with an excellent hemostasis by coagulation and cut simultaneously, with less heat production and surrounding tissue damage, and finally less post-surgical pain.

\section{REFERENCES}

[1] David S. Leonard, FRCSI, Conrad Timon, MB, FRCSORL, Evaluation of the ultrasonic dissector in head and neck surgery, Operative Techniques in Otolaryngology (2008) 19, 59-66.

[2] Intelligent Ultrasonic Energy instructive, Ethicon Johnson \& Johnson Medical, Instructions.

[3] Thomas Carroll, Keith Ladner, Arlen D. Meyers, Alternative Surgical Dissection Techniques, Otolaryngol Clin N Am 38 (2005) 397-411.

[4] Giancarlo Tirelli, Giulia Carolina Del Piero, Fiorella Perrino, Ultracision Harmonic Scalpel in oral and oropharyngeal cancer resection, Journal of Cranio-Maxillo-Facial Surgery 42 (2014) 544-547

[5] Arulalan Mathialagan, Roshan K. Verma, Naresh K. Panda, Comparison of spinal accessory dysfunction following neck dissection with harmonic scalpel and electrocautery - A randomized study, Oral Oncology 61 (2016) 142-145.

[6] Luca Revelli, Gianfranco Damiani, Caterina Bianca Neve Aurora Bianchi, Serafino Vanella, Walter Ricciardi, Marco Raffaelli, Celestino Pio Lombardi, Complications in thyroid surgery. Harmonic Scalpel, Harmonic Focus versus Conventional Hemostasis: A meta-analysis, International Journal of Surgery 28 (2016) 2232.

[7] Roberto Ruggiero, Ludovico Docimo, Salvatore Tolone, Maurizio De Palma, Mario Musella, Angela Pezzolla, Adelmo Gubitosi, Domenico Parmeggiani, Raffaele Pirozzi, Simona Gili,
Simona Parisi, Antonio D'Alessandro, Giovanni Docimo, Effectiveness of an advanced hemostatic pad combined with harmonic scalpel in thyroid surgery. A prospective study, International Journal of Surgery 28 (2016) 1721.

[8] Fan S, Zhang D-m, Chen W-1, Endoscopyassisted resection of benign lesions on the base of the tongue via the transoral approach using a harmonic scalpel, Journal of Oral and Maxillofacial Surgery (2017), doi: 10.1016/j.joms.2017.03.002.

[9] Hakan Bulus, Adnan Tas, Ali Coskun, Metin Kucukazman, Evaluation of two hemorrhoidectomy techniques: Harmonic scalpel and Ferguson's with electrocautery, Asian Journal of Surgery (2014) 37, 20-23.

[10] Emanuele Ferri, Enrico Armato, Giacomo Spinato, Roberto Spinato, Focus Harmonic Scalpel Compared to Conventional Haemostasis in Open Total Thyroidectomy: A Prospective Randomized Trial, International Journal of Otolaryngology Volume 2011, 7.

[11] Roshan K. Verma, Arulalan Mathiazhagan, Naresh K. PandaNeck dissection with harmonic scalpel and electrocautery? A randomized study. Auris Nasus Larynx (2016), 11.004.

[12] Naseem Waraich, Javed Ahmed, Farhan Rashid, David Mulvey, Paul Leeder, Syed Y. Iftikhar, Is harmonic scalpel an effective tool for oesophagectomy?, International Journal of Surgery 7 (2009) 330-333.

[13] Jawad Kadhim S. Al-Dhahiry, Husam Majeed Hameed, Total thyroidectomy: Conventional Suture Ligation technique versus sutureless techniques using Harmonic Scalpel or Maximum, Annals of Medicine and Surgery 5 (2016) 29-34.

[14] Yoon Woo Koh, MD, Ph.D., Eun Chang Choi, Robotic Approaches to the Neck, Otolaryngol Clin N Am 47 (2014) 433-454.

[15] Eric Goudie, Mehdi Tahiri, Moishe Liberman, Present and Future Application of Energy Devices in Thoracic Surgery, Thorac Surg Clin 26 (2016) 229-236.

[16] Russell B. Smith, Andrew Coughlin, Thyroidectomy Hemostasis, Otolaryngol Clin N Am 49 (2016) 727-748.

Citation: Carrillo Rivera Jorge Arnulfo et al. Curve Sagittal Mentoplasty, Description of the Technique and Evaluation of the Neurosensory Response. Case Series. ARC Journal of Surgery.2018;4(2) :18-21. doi: dx.doi.org/ 10.20431/2455-572X. 0402004.

Copyright: (c) 2018 Authors. This is an open-access article distributed under the terms of the Creative Commons Attribution License, which permits unrestricted use, distribution, and reproduction in any medium, provided the original author and source are credited. 